https://doi.org/10.15407/ujpe66.4.293

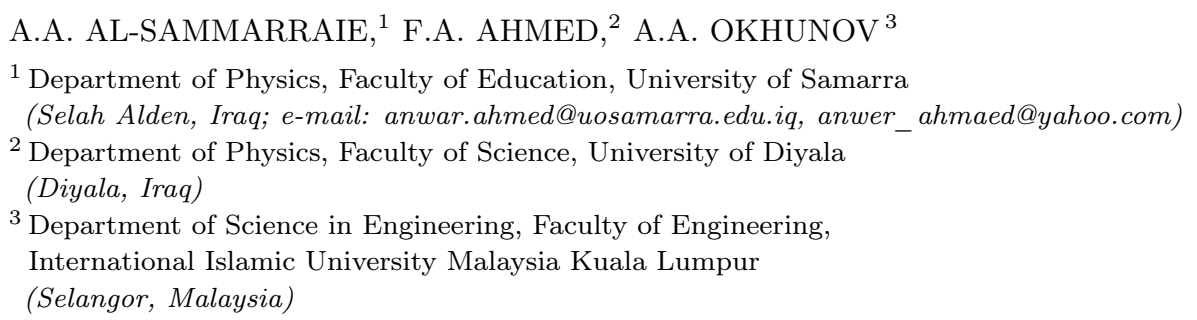

\title{
LARGE SCALE SHELL MODEL CALCULATIONS OF THE NEGATIVE-PARITY STATES STRUCTURE IN ${ }^{24} \mathrm{Mg}$ NUCLEUS
}

\begin{abstract}
The negative-parity states of ${ }^{24} \mathrm{Mg}$ nucleus are investigated within the shell model. We are based on the calculations of energy levels, total squared form factors, and transition probability using the $p$-sd-pf (PSDPF) Hamiltonian in a large model space $(0+1) \hbar \omega$. The comparison between the experimental and theoretical states showed a good agreement within a truncated model space. The PSDPF-based calculations successfully reproduced the data on the total squared form factors and transition probabilities of the negative-parity states in ${ }^{24} \mathrm{Mg}$ nucleus. These quantities depend on the one-body density matrix elements that are obtained from the PSDPF Hamiltonian. The wave functions of radial one-particle matrix elements calculated with the harmonic-oscillator potential are suitable to predict experimental data by changing the centerof-mass corrections.
\end{abstract}

Keywords: PSDPF, negative-parity states, electron scattering, shell model, form factors, ${ }^{24} \mathrm{Mg}$.

\section{Introduction}

Recently, the nuclei with $N=Z$ have received a significant attention in many shell model calculations [1-6]. The importance of these nuclei appears from the study of pairing correlations between fermions, as well as the excited states which could be isoscalar with $T=0$ or isovector with $T=1$. The study of these excited states represents an effective manner to understand the behavior of many-body quantum systems. ${ }^{24} \mathrm{Mg}$ nucleus is one of these nuclei with distinctive properties and has the sufficient number of nucleons for shell model calculations and for the ex-

(C) A.A. AL-SAMMARRAIE, F.A. AHMED, A.A. OKHUNOV, 2021

ISSN 2071-0194. Ukr. J. Phys. 2021. Vol. 66, No. 4 hibition of collective excitations associated with large, prolate deformations $[7,8]$.

${ }^{24} \mathrm{Mg}$ has $Z=12$ and $N=12$ and represents a rich testing ground for microscopic descriptions of the exotic nuclei with a shell structure based on cross-shells configurations [9]. Studying the excited states of this nucleus with the use of the shell model clarifies the various configurations of nucleons in the main valence $s d$ shell for the positive-parity states, and the intruder excitations of the negative-parity states with crossshell configurations in $p$ or/and $f p$ shells.

The negative-parity states, named intruder states, accrue due to the promotion of one nucleon from the $p$ to $s d$ shell for nuclei near ${ }^{16} \mathrm{O}$, or from $s d$ to $f p$ shell for nuclei close to ${ }^{40} \mathrm{Ca}[10] .{ }^{24} \mathrm{Mg}$ nucleus is po- 
sitioned in the middle of the $s d$ shell, where there is a competition between the two types of transitions. Limited theoretical studies have been conducted concerning these nuclear states designated for individual angular momentum $J$ values [9-13], despite a wide range of experimental data.

The effective interaction used in this work should include the full model space $p-s d-p f$. The PSDPF interaction [13] was developed to describe the positiveand negative-parity states in $s d$-shell nuclei within the full $p-s d-p f$ model space, allowing nucleons to move from $p$ to $s d$ or/and from $s d$ to $p f$ shells with a ${ }^{4} \mathrm{He}$ core. Recently, many shell model calculations have shown the remarkable results using this interaction [14-17].

The electromagnetic transitions can give an important information on the nuclear structure. The scattering form factors describe the electromagnetic properties of the nucleus. Inelastic scattering form factors represent a striking feature which can be investigated theoretically and experimentally [18]. From this, we can verify the precision of the PSDPF interaction. In this work, the negative-parity states, electromagnetic transitions, and inelastic scattering form factors are calculated using the PSDPF interaction for ${ }^{24} \mathrm{Mg}$ nucleus.

\section{Results and Discussions}

The calculations were performed using the OXBASH code for Windows [19]. The code uses the $M$-scheme Slater determinant basis, a projection technique, and wave functions with definite angular momentum $J$ and isospin $T$. The PSDPF interaction includes four main parts: CK [20], USDB [21], and PSDT [22] for the $p, s d$ and $p-s d$ shells, respectively, while IOKIN [23] for the $p f$ and $s d-p f$ shells. The values of crossshells have been found from the fitting procedure with experimental data [13]. The single-particle energies of the $p-s d-p f$ model space are 2.27, 1.63, $5.2588,2.5767,8.0649,15.7,15.9102,16.0102$, and $18.8102 \mathrm{MeV}$ for shells $1 p_{1 / 2}, 1 p_{3 / 2}, 1 d_{5 / 2}, 2 s_{1 / 2}$, $1 d_{3 / 2}, 1 f_{7 / 2}, 2 p_{1 / 2}, 2 p_{3 / 2}$, and $1 f_{5 / 2}$, respectively.

\subsection{Energy levels}

The number of valance particles of ${ }^{24} \mathrm{Mg}$ nucleus has an advantage, which is the capability to occupy the full $p-s d-p f$ model space in order to calculate the negative-parity states. On the other hand, the valance particles make a huge number of possible configurations for the nucleons in this model space. These configurations require a large memory space and a long calculation time. In order to overcome these obstacles, the $p-s d-p f$ model space has been truncated by assuming that the configuration will occur in $p-s d$ shells.

The wave function of the ground state has been calculated with ${ }^{16} \mathrm{O}$ core, where a good agreement has been found with experimental data for a positiveparity state using USDB as in [24]. The comparisons between experimental and calculated result are shown in Fig. 1. The experimental results provided four excitation states with $J=1^{-}$, and these states are well reproduced throughout the shell model calculations with energy greater than $11 \mathrm{MeV}$. The noticeable gaps between theoretical states made it possible to assign them to experimental states. Therefore, the sequences of the experimental states have been adopted with respect to the energy values of the theoretical states.

According to the theoretical results, the states with $J=1^{-}$appeared with a structure mostly related to a hole in the $1 p$ shells, see Table 1 , with a major contribution for the shells $1 p_{3 / 2}$ and $1 d_{5 / 2}$. The occupation probability increases for shell $1 p_{3 / 2}$, as the energy increases, and decreases for $1 d_{5 / 2}$ shell.

The experimental spectrum of ${ }^{24} \mathrm{Mg}$ nucleus shows one state with $J=2^{-}$at an energy of $8.864 \mathrm{MeV}$ and the isospin $T=0$. This state has been predicted with an energy of $9.189 \mathrm{MeV}$ at the second sequence. The first and third theoretical states (sequence 1 and 3 ) are predicted with energies of 6.326 and $9.444 \mathrm{MeV}$, respectively. Depending on the energy value, the second sequence state with an energy of $9.189 \mathrm{MeV}$ is compatible with the experimental one. The average number of particles in the $p-s d$ model space appeared similar to that of $J=1^{-}$, as presented in Table 1 .

The excited states with $J=3^{-}$attracted a wide attention during many previous works, especially at 7.616 and $8.357 \mathrm{MeV}[11,13,14,25]$. In this work, the total spectrum of these states has presented in order to obtain a clear perception about the compatibility of these states attributed to theoretical calculations. The PSDPF calculations showed the energies of the first and second $J=3^{-}$states at 7.394 and $7.750 \mathrm{MeV}$, respectively, as compared to the experimental values of 7.616 and $8.357 \mathrm{MeV}$, respectively, an acceptable value was found for the first state. The

ISSN 2071-0194. Ukr. J. Phys. 2021. Vol. 66, No. 4 

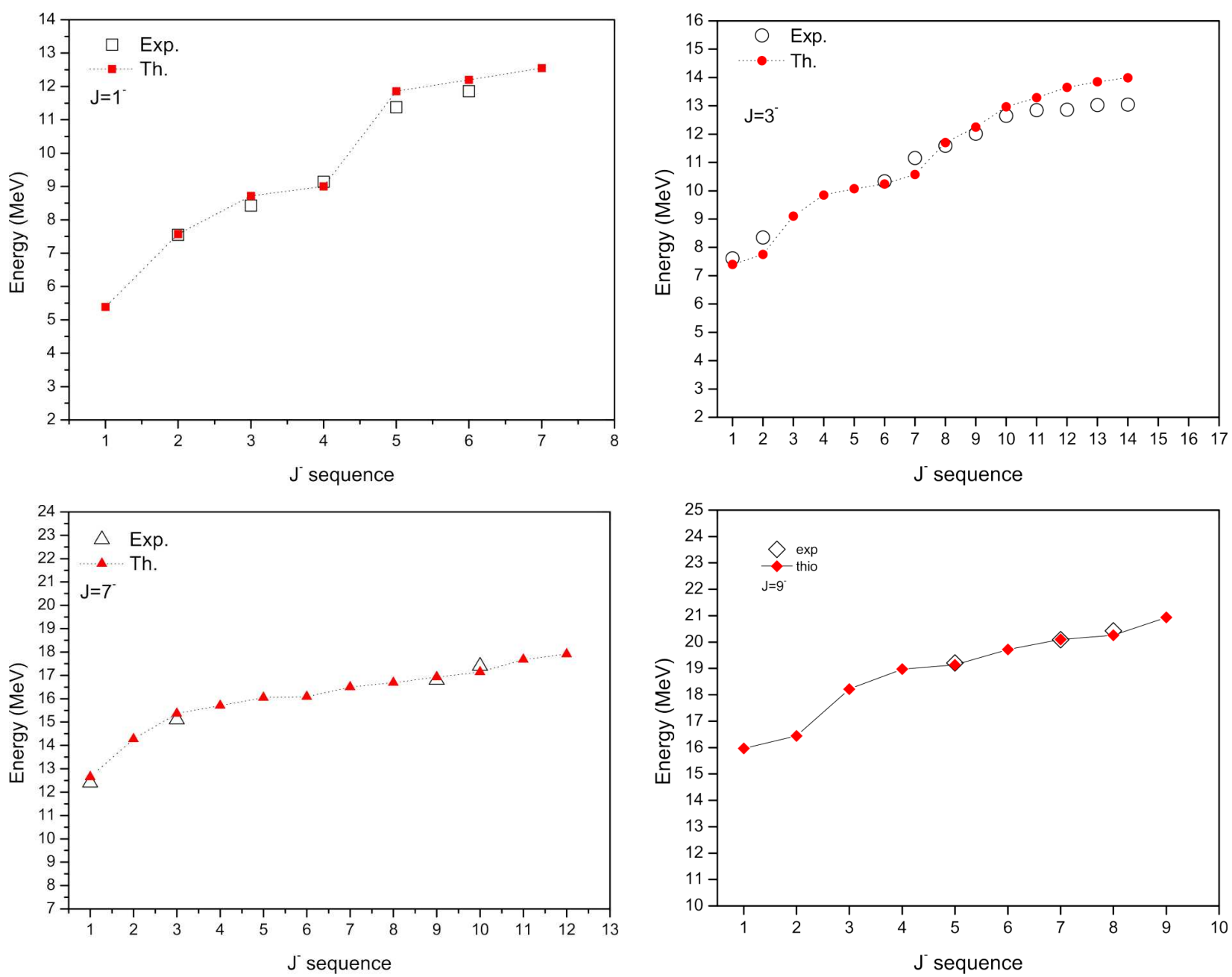

Fig. 1. (Color online) Comparison between calculated and experimental excitation energies of the $J=1^{-}, 3^{-}, 7^{-}$, and $9^{-}$ states for ${ }^{24} \mathrm{Mg}$ nucleus. The experimental data are taken from [9, 11, 26, 27]. The theoretical $J^{-}$sequences have been adopted according to the closest energy from the theoretical results using the PSDPF interaction

second theoretical state appeared lower than the experimental one. Present calculations have shown that the first and second $3^{-}$states have an almost pure $1 p^{-1}$ shell configuration, see Table 1 , and are the same as found in [11]. In the top-right in Fig. 1, the acceptable agreement between theoretical and experimental states continue until the state with eleventh sequences. For higher states, there is a clear incompatibility.

The comparison between theoretical and experimental levels showed a disagreement for the states with $J \geq 4^{-}$, when the assumption of a truncated model space had used. In order to fix this regression, the PSDPF model space was extended to permit one particle to move to the $f_{7 / 2}$ shell. This procedure has shown a marked improvement in PSDPF calculations, see Fig. 1 and Table 1 . The state of $J=5^{-}$has been found experimentally at an energy of $10.02 \mathrm{MeV}[11,26]$. According to the present calculation, this state is clearly associated with a particle in the $f p$ shell, and it has been predicted at an energy of $11.10 \mathrm{MeV}$. The recent negative-parity states of ${ }^{24} \mathrm{Mg}$ nucleus have been identified in [9] with $J=7^{-}$and $9^{-}$. The comparison with the present calculation is shown in Fig. 1. It is worth noting that these states show lower sequences, as compare with the experimental data. The last $7^{-}$state has been identified experimentally at an energy of $19.98 \mathrm{MeV}$ 
Table 1. The calculated negative-parity states compared with the experimental energy levels using the occupancy probability for ${ }^{24} \mathrm{Mg}$

\begin{tabular}{|c|c|c|c|c|c|c|c|c|c|}
\hline \multirow{2}{*}{$J_{i}^{-}$} & \multirow{2}{*}{$E_{\text {theor }}, \mathrm{KeV}$} & \multirow{2}{*}{$E_{\text {exp }}, \mathrm{KeV}$} & \multicolumn{6}{|c|}{ Average number of particles in each $j$-level } & \multirow{2}{*}{ P.E \% } \\
\hline & & & $1 P_{1 / 2}$ & $1 P_{3 / 2}$ & $1 D_{5 / 2}$ & $2 S_{1 / 2}$ & $1 D_{3 / 2}$ & $1 F_{7 / 2}$ & \\
\hline $1_{2}^{-}$ & 7576 & $7555[26,27]$ & 3.718 & 7.281 & 6.920 & 0.908 & 1.171 & 0.000 & 0.278 \\
\hline $3_{1}^{-}$ & 7394 & $7616[26,27]$ & 3.695 & 7.304 & 6.809 & 1.030 & 1.159 & 0.000 & 2.915 \\
\hline $3_{2}^{-}$ & 7750 & $8357[26,27]$ & 3.395 & 7.604 & 6.992 & 0.837 & 1.169 & 0.000 & 7.273 \\
\hline $1_{3}^{-}$ & 8727 & $8437[26,27]$ & 3.493 & 7.506 & 6.501 & 1.337 & 1.161 & 0.000 & 3.437 \\
\hline $2_{2}^{-}$ & 9189 & $8864[26,27]$ & 3.615 & 7.384 & 6.223 & 1.081 & 1.694 & 0.000 & 3.667 \\
\hline $1_{4}^{-}$ & 9007 & $9145[26,27]$ & 3.690 & 7.309 & 6.216 & 1.239 & 1.543 & 0.000 & 1.509 \\
\hline $5_{1}^{-}$ & 11103 & $10027[26,27]$ & 3.990 & 7.776 & 5.301 & 1.197 & 0.948 & 0.776 & 10.731 \\
\hline $3 \frac{-}{6}$ & 10235 & $10332[11,26,27]$ & 3.610 & 7.389 & 6.733 & 1.104 & 1.542 & 0.000 & 0.939 \\
\hline $1_{5}^{-}$ & 11862 & $11389[26,27]$ & 3.665 & 7.334 & 6.288 & 1.168 & 1.162 & 0.000 & 4.153 \\
\hline $33_{7}^{-}$ & 10579 & $11162[26,27]$ & 3.491 & 7.508 & 6.238 & 1.507 & 1.264 & 0.000 & 5.223 \\
\hline $1_{6}^{-}$ & 12283 & $11864[26,27]$ & 3.506 & 7.493 & 6.587 & 1.274 & 1.137 & 0.000 & 3.532 \\
\hline $7_{1}^{-}$ & 12749 & $12441[9]$ & 3.771 & 8.000 & 5.165 & 1.324 & 0.912 & 0.827 & 1.672 \\
\hline $3_{8}^{-}$ & 11707 & $11596[26,27]$ & 3.365 & 7.635 & 6.978 & 0.969 & 1.053 & 0.000 & 0.716 \\
\hline $3_{9}^{-}$ & 12263 & $12015[26,27]$ & 3.593 & 7.407 & 6.992 & 1.035 & 0.974 & 0.000 & 0.558 \\
\hline $3_{10}^{-}$ & 12966 & $12758[26,27]$ & 3.683 & 7.318 & 6.839 & 1.183 & 0.978 & 0.000 & 0.079 \\
\hline $3_{11}^{-}$ & 13289 & $12845[26,27]$ & 3.485 & 7.516 & 6.363 & 1.537 & 1.100 & 0.000 & 0.070 \\
\hline $3_{12}^{-}$ & 13652 & $12861[26,27]$ & 3.442 & 7.558 & 6.372 & 1.445 & 1.183 & 0.000 & 2.302 \\
\hline $3_{13}^{-}$ & 13851 & $13027[26,27]$ & 3.375 & 7.626 & 6.227 & 1.455 & 1.318 & 0.000 & 3.585 \\
\hline $3_{14}^{-}$ & 13991 & $13050[26,27]$ & 3.460 & 7.540 & 6.352 & 1.400 & 1.248 & 0.000 & 4.621 \\
\hline $4_{9}^{-}$ & 13215 & $13056[26,27]$ & 3.184 & 8.000 & 5.893 & 1.212 & 1.527 & 0.184 & 1.218 \\
\hline $7_{3}^{-}$ & 15365 & $15180[9]$ & 3.580 & 8.000 & 5.367 & 1.058 & 1.331 & 0.662 & 1.219 \\
\hline $7_{9}^{-}$ & 16927 & $16920[9]$ & 3.235 & 8.000 & 5.932 & 1.366 & 1.116 & 0.349 & 0.035 \\
\hline $7_{11}^{-}$ & 17683 & $17440[9]$ & 3.329 & 8.000 & 5.350 & 1.551 & 1.341 & 0.427 & 1.393 \\
\hline $9_{5}^{-}$ & 19133 & $19210[9]$ & 3.835 & 8.000 & 4.992 & 1.242 & 1.039 & 0.889 & 0.401 \\
\hline $7_{28}^{-}$ & 19947 & $19980[9]$ & 3.348 & 8.000 & 5.684 & 1.214 & 1.301 & 0.450 & 0.165 \\
\hline $9_{7}^{-}$ & 20099 & $20090[9]$ & 3.679 & 8.000 & 5.341 & 1.432 & 0.805 & 0.741 & 0.045 \\
\hline $9_{8}^{-}$ & 20269 & $20420[9]$ & 3.856 & 8.000 & 5.160 & 0.926 & 1.149 & 0.906 & 0.788 \\
\hline
\end{tabular}

P.E. is the percentage error which is calculated from P.E. $=\left|\frac{E_{\exp }-E_{\text {theor }}}{E_{\exp }}\right|$.

(not shown in Fig. 1). The corresponding theoretical state is predicted at sequence $28^{\text {th }}$ with an energy of 19.94 MeV. The contribution of nucleon excitations to the $f p$ shell is very clear for the calculations of $7^{-}$ and $9^{-}$states, which is presented in Table 1 .

\subsection{Electromagnetic transitions and form factors}

Many nuclear features can can be studied theoretically and experimentally through their electromagnetic properties. On the other hand, the transition rates represent a sensitive test for the most modern effective interactions [28]. In shell model calculations, the electromagnetic transition depends upon the reduced nuclear matrix elements, and this provided an accurate test for the precision of the Hamiltonian used. The reduced probabilities of electric octupole transitions have been measured experimentally for the transition from the ground state to the $J=3^{-}$ at energies of 7.616 and $8.357 \mathrm{MeV}$ [29]. The good agreement between theoretical and experimental values supports our presumed configurations presented in Table 2. The values of the effective charges which are used in this calculations are the same as those in $[14,30]$. 
H. Zarek et al. [29] studied experimentally and theoretically the inelastic electron scattering form factors for the stronger transitions to negative-parity states in ${ }^{24} \mathrm{Mg}$. The total squared form factors were measured for electron energies of $90-280 \mathrm{MeV}$ and scattering angles of $90^{\circ}$ and $160^{\circ}$. The total squared form factor are given by[31]:

$F^{2}(q, i, f)=F_{c}^{2}(q, i, f)+$

$+\left[0.5+\tan ^{2}\left(\frac{\theta}{2}\right)\right] F_{T}^{2}(q, i, f)$,

where $F_{c}^{2}(q, i, f)$ is the squared longitudinal form factor, and $F_{T c}^{2}(q, i, f)$ is the squared transverse (electric or magnetic) form factor for the transition between the initial state $i$ and final state $f$ with threemomentum transfer $(q), \theta$ is the scattering angle of an electron. Both $F_{c}$ and $F_{T}$ can be found from the relations [31]

$$
\begin{aligned}
& F^{2}(X L, q, f, i)= \\
& =N_{p} G_{c m}^{2}(q) \Sigma_{t_{z}, x} W_{f s}\left(X L x, q, f, i, t_{z}\right)^{2}, \\
& N_{p}=\frac{4 \pi}{z^{2}\left(2 J_{i}+1\right)}, \\
& G_{c m}(q)=e^{\left(\frac{b^{2} q^{2}}{4 A}\right)},
\end{aligned}
$$

where $W_{f s}\left(X L x, q, f, i, t_{z}\right)$ are reduced matrix elements calculated taking the finite-size of nucleons into account, $x$ indicates the magnetic $(m)$ and convection current $(c)$ contributions for the electric $(X=E)$ and magnetic $(X=M)$ form factors, and $X=C$ in the case of the Coulomb form factor, $A$ and $Z$ are the atomic mass and atomic number of the target nucleus, respectively, $G_{c m}$ is the center-of-mass correction, and $b$ is the oscillator length parameter chosen to reproduce the root mean square radius of the nucleus (rms). The details of calculations of $F_{c}$ and $F_{T}$ from $W_{f s}\left(X L x, q, f, i, t_{z}\right)$ are presented in [31-33]. The reduced matrix elements $W_{f s}\left(X L x, q, f, i, t_{z}\right)$ had been calculated as follows[32]:

$$
\begin{aligned}
& W_{f s}\left(X L x, q, f, i, t_{z}\right)= \\
& =w_{f s}\left(X \lambda x, q, f, i, t_{z}\right) \frac{g_{f s}\left(X x, q, t_{z}\right)}{g\left(X x, t_{z}\right.},
\end{aligned}
$$

where $w_{f s}\left(X \lambda x, q, f, i, t_{z}\right)$ is the point-nucleon reduced matrix elements with multipolarity $\lambda$, and
$g\left(X x, t_{z}\right)$ is the free-nucleon $g$ factors, with $X x=$ $=M c, E c$, or $C ; g\left(X x, t_{z}\right)=g_{l}\left(t_{z}\right)$ and for $X x=$ $=M m$ or $E m ; g\left(X x, t_{z}\right)=g_{s}\left(t_{z}\right), g_{f s}\left(X x, q, t_{z}\right)$ is the experimental form factors for free nucleons [34]. The multiparticle form factors $w\left(X \lambda x, q, f, i, t_{z}\right)$ are given as [32]:

$w_{f s}\left(X \lambda, q, f, i, t_{z}\right)=\Sigma_{k, k^{\prime}} \operatorname{OBDM}\left(\lambda, k, k^{\prime}, f, i, t_{z}\right) \times$ $\times w_{f s}\left(X \lambda, q, k, k^{\prime}, t_{z}\right)$,

where $X$ represents $C, M c, M m, E c$, or $E m$. The sum $\left(k, k^{\prime}\right)$ runs over all pairs of single-particle states in the model space.

The one-body density-matrix elements $\operatorname{OBDM}\left(\lambda, k, k^{\prime}, f, i, t_{z}\right)$ are calculated in the isospin formalism. In the present work, the calculations of OBDM are performed in the $p-s d-p f$ model space using the PSDPF interaction with valence (active) particles restricted as presented in the energy state calculations.

The electromagnetic transitions conserve the parity. In order to determine the type of transition being electric or magnetic according to the multipolarity $\lambda,\left|J_{i}^{\pi}-J_{f}^{\pi}\right| \leq \lambda \leq J_{i}^{\pi}-J_{f}^{\pi}$, the selection rule follows $\pi_{i} \pi_{f} \pi_{o}=+1$, where $\left(\pi_{i}\right)$ is the parity of the initial state, $\left(\pi_{f}\right)$ is the parity of the final state, and $\pi_{o}=(-1)^{\lambda}$ for the electric transition or $\pi_{o}=(-1)^{\lambda+1}$ for the magnetic transition [31].

The transitions from the ground state $J_{i}^{\pi}=0^{+}$ to $J_{f}^{\pi}=1^{-}$have a multipolarity equal 1 . According to the selection rule above, it will be an electric transition. Work [29] presents the experimental data on the squared form factor for the transition from the ground sate $\left(J_{i}^{\pi}=0^{+}\right)$to the excited state with $J_{f}^{\pi}=1^{-}$at energies of 7.553 and $8.438 \mathrm{MeV}$ in ${ }^{24} \mathrm{Mg}$. In Fig. 2, A, the theoretical and experimental squared form factor are compared for the transition to the $J^{\pi}=1^{-}$at an energy of $7.555 \mathrm{MeV}$ for the electron scattering angles $\theta=90^{\circ}$ and $160^{\circ}$. The theoretical squared form factor was calculated with the

Table 2. Calculated and experimental B(E3) values from the ground state to the $3^{-}$in ${ }^{24} \mathrm{Mg}$. The experimental values taken from [29].

\begin{tabular}{|c|c|c|c|}
\hline $\begin{array}{c}E_{\exp }, \\
\mathrm{MeV}\end{array}$ & $\begin{array}{c}E_{\mathrm{theor}}, \\
\mathrm{MeV}\end{array}$ & $\begin{array}{c}B(E 3) \uparrow_{\exp } e^{2}, \\
\mathrm{fm}^{6}\end{array}$ & $\begin{array}{c}B(E 3) \uparrow_{\text {theor }} e^{2}, \\
\mathrm{fm}^{6}\end{array}$ \\
\hline 7.616 & 7.394 & 562 & 542 \\
8.357 & 7.750 & 1580 & 1476 \\
\hline
\end{tabular}



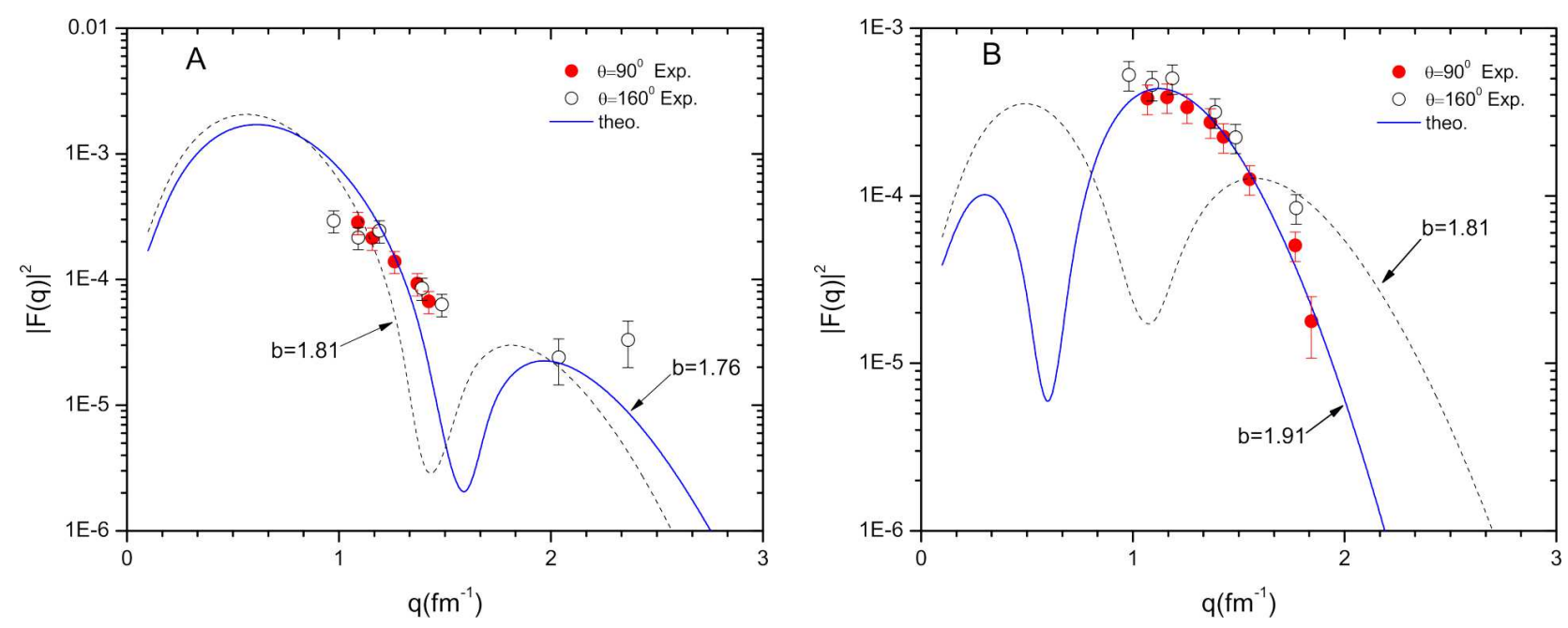

Fig. 2. (Color online) Total squared form factor for the transition from the ground state to the $J^{\pi}=1^{-}(7.555 \mathrm{MeV})$ and $J^{\pi}=1^{-}(8.437 \mathrm{MeV})$ in ${ }^{24} \mathrm{Mg}$ obtained with the use of the harmonic oscillator potential and the PSDPF Hamiltonian (A) and (B), respectively. The experimental data are taken from [29]

electron scattering angle $\theta=90^{\circ}$. The comparison based on the $F_{\theta=90^{\circ}}^{2}(q, i, f) \approx F_{\theta=160^{\circ}}^{2}(q, i, f)$ can be considered acceptable [29]. The energy of the 7.555$\mathrm{MeV}$ state was calculated to be $7.576 \mathrm{MeV}$ at the second sequence. The best prediction of the experimental data is getting, when the center-of-mass correction is employed as a correction factor. The center-of-mass correction $G_{c m}$ was suggested by Tassie et al. [35] in view of the motion of the nucleus mass center at the scattering of high-energy electrons. We have change the $b$ value for $G_{c m}$ and keep the default value in the a harmonic oscillator potential. The best agreement with experimental results has been found, if we used $b=1.76 \mathrm{fm}$ and the new effective charges, $1.36 e$ for protons and $0.45 e$ for neutrons $[14,30]$.

Figure 2, B shows the total squared form factor for the transition from the ground to $J^{\pi}=1^{c}$ state with an energy of $8.437 \mathrm{MeV}$. The shell model predicts this state at sequence 3 with an energy of $8.727 \mathrm{MeV}$. The present calculations of the total squared form factor have shown a good agreement with experimental data [29]. The theoretical form factor values have two peaks at $q=0.3$ and $1.6 \mathrm{fm}^{-1}$, and only the latter was observed experimentally. The calculations of form factors are performed by using effective charges and $b=1.91 \mathrm{fm}$.

The squared form factors for the states $J^{\pi}=$ $=3^{-}$at energies 7.616 and $8.357 \mathrm{MeV}$ are plotted in Fig. 3, A and B, respectively. The transition E3 from the ground state to the $7.616-\mathrm{MeV}$ state is shown in Fig. 3, A. The calculated values is less than the experimental data, when the default parameters of the OXBASH program are used. The oscillator length parameter has changed to $1.92 \mathrm{fm}$ which is making the theoretical results more close to experimental data.

Experimental data of the transition E3 from the ground state to the the $8.357-\mathrm{MeV}$ state show two peaks at $q=0.98$ and $2.38 \mathrm{fm}^{-1}$ in Fig. 3, B. The difference between the two modes of the transition for $3_{1}^{-}$and $3_{2}^{-}$indicates that $3_{2}^{-}$has a larger transition radius than $3_{1}^{-}$, which testifies that these states are indeed different $[29,36]$. Branford et al. [36] have suggested that the states $J^{\pi}=3^{-}$and $5^{-}$at energies 8.357 and $10.028 \mathrm{MeV}$ are members of a $K^{\pi}=0^{-}$ band. The state $3^{-}$at an energy of $7.617 \mathrm{MeV}$ is the bandhead of a $K^{\pi}=3^{-}$. The theoretical squared form factors have shown a good precision for the first peak, when the $b=2.3 \mathrm{fm}$, while the second peak did not appear theoretically.

A comparison between PSDPF calculations and the experimental data on the energy values for the state $5^{-}$have been shown a difference of $\sim 1 \mathrm{MeV}$, see Table 1 . The form factor calculations depend on the eigenvectors of the Hamiltonian (particle wave functions). A comparison between the theoretical and experimental values of the total squared form factor for the transition from the ground state to the state $5^{-}$ 

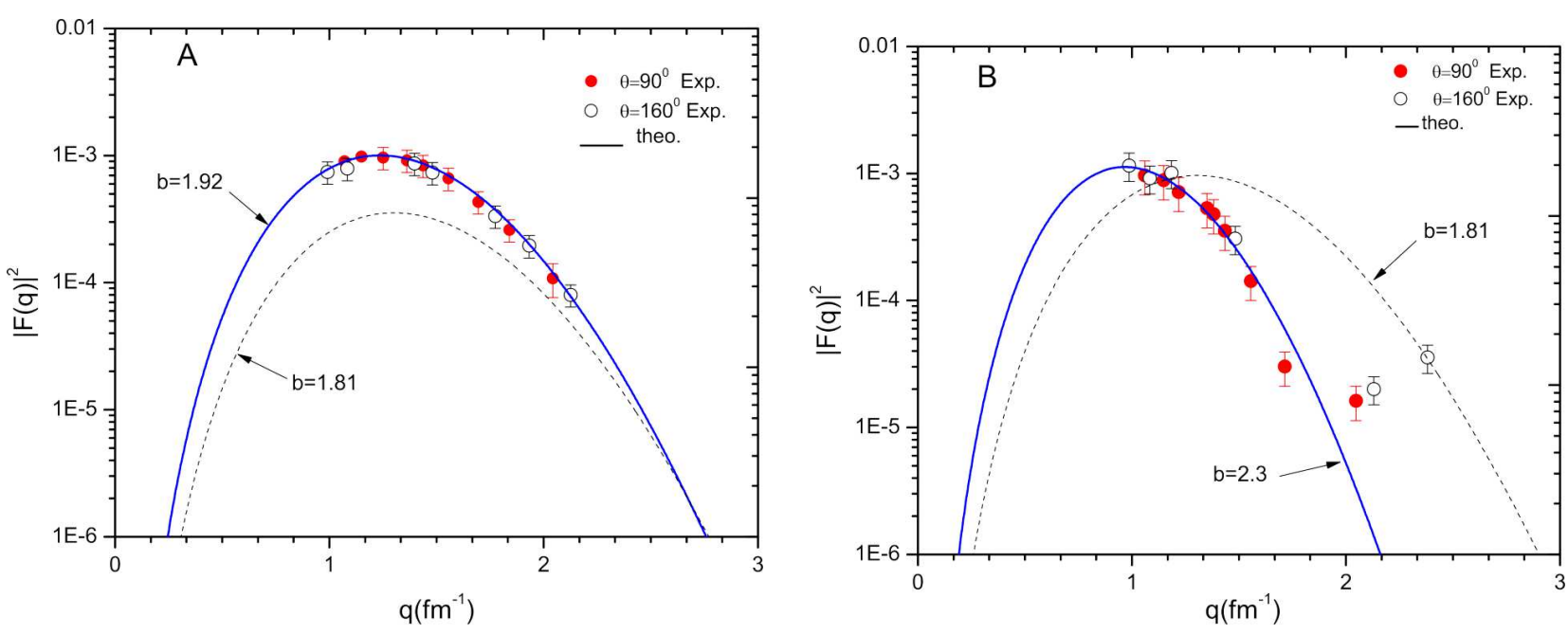

Fig. 3. (Color online) Total squared form factor for the transition from the ground state to the $J^{\pi}=3^{-}(7.616 \mathrm{MeV})$ and $J^{\pi}=3^{-}(8.357 \mathrm{MeV})$ states in ${ }^{24} \mathrm{Mg}$ obtained with the use of the harmonic oscillator potential and the PSDPF Hamiltonian; (A) and (B), respectively. The experimental data are taken from [29]
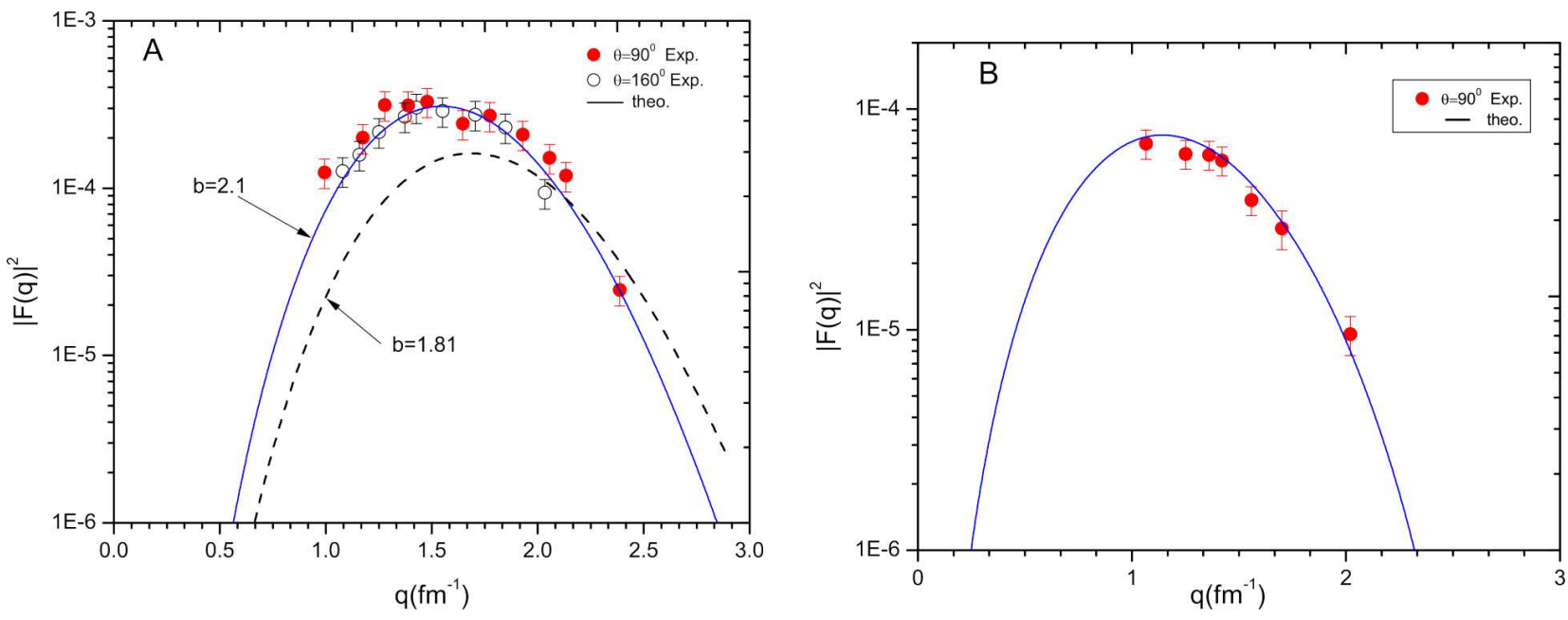

Fig. 4. (Color online) Total squared form factor for the transition from the ground state to the $J^{\pi}=5^{-}(10.027 \mathrm{MeV})$ and $J^{\pi}=2^{-}, T=1(12.67 \mathrm{MeV})$ states in ${ }^{24} \mathrm{Mg}$ obtained with the use of the harmonic oscillator potential and the harmonic oscillator potential and the PSDPF Hamiltonian; (A) and (B), respectively. The experimental data are taken from [29]

is shown in Fig. 4, A. The oscillator length parameter $b=2.1 \mathrm{fm}$ and the effective charge for a proton $e_{p}=1.0$ are used to give matching with experimental data.

Level $J=2$ at an energy of $12.669 \mathrm{MeV}$ has been presented without isospin and parity in $[26,27]$. H. Zarek et al. [29] observed an energy level at $12.650 \pm 0.05 \mathrm{MeV}$ with $J=2^{-}$and isospin $T=1$. Johnston et al. [37] and Lawergren et al. [38] have shown that this state has an energy of $12.67 \mathrm{MeV}$,
$J=2^{-}$, and isospin $T=1$. The nearest theoretical energy states $J=2^{-}$with $T=0$ and $T=1$ withe use of the PSDPF interaction are $12.725 \mathrm{MeV}$ at $8^{\text {th }}$ sequence and $12.809 \mathrm{MeV}$ at first sequence, respectively (not shown in Table 1). H. Zarek et al. [29] measured the squared form factor for the transition from the ground state to the $(12.650 \pm 0.05)-\mathrm{MeV}$ state with $J=2^{-}$and isospin $T=1$. The comparison between theoretical and experimental squared form factor will give a preference to the most accurate theoretical 
state. According to the multipolarity selection rule, this transition must be a magnetic quadrupole transition M2 and depend on the scattering angle $\theta$ of an electron, see Eq. (1). The OXBASH program considered $\theta=90^{\circ}$ as a default value for the scattering angle. Therefore, the experimental date for $\theta=90^{\circ}$ are adopted in comparison. The calculations show that the maximum value of the squared form factor for $J=2^{-}, T=1$ higher than $J=2^{-}, T=0$ by almost $10^{2}$, and the higher values are closer to the experimental values. As shown in Fig. 4, B, the matching between theoretical and experimental results has found by using oscillator length parameter $b=1.62 \mathrm{fm}$, and effective nucleon $g$ factors as $g_{s}^{\pi}=5.05, g_{s}^{v}=-3.0$, $g_{l}^{\pi}=1, g_{l}^{v}=0[18,30]$, whereas the magnetic form factors depend on the nucleon $g$ factors.

\section{Conclusions}

In the present work, the intruder negative-parity states of ${ }^{24} \mathrm{Mg}$ nucleus have been studied theoretically using the large model space and the PSDPF Hamiltonian. The calculations include energy levels, reduced transition probabilities, and total squared form factor. By comparison with experimental data, it has been found that the states with $J \leq 3^{-}$ can be obtained in a reasonable agreement using a truncated model space and configurations $\left(1 p_{1 / 2}\right.$, $\left.1 p_{3 / 2}\right)^{-1}$, while for those with $J \leq 4^{-}$by allowing one nucleon to move to the $1 f_{7 / 2}$ shell. The theoretical results for the states $J=3^{-}$have shown that these states have the critical situation, since the spin and energy values affect data on the distribution of nucleons in the shells. The results on electromagnetic transitions support our predictions for the transition probabilities and total squared form factor. We have shown that the results on total squared form factors obtained with the use of the PSDPF Hamiltonian with harmonic oscillator potential in the $p-s d-p f$ model space are in a reasonable agreement with experimental data for the overall states in the momentum transfer interval $0<q<3 \mathrm{fm}^{-1}$. The overlaps between the theoretical value and experimental data have been founded by changing the center-of-mass correction values. These changes have been made by employing the oscillator length parameter $b$ as a free parameter in calculating the center-of-mass correction values and keep the default value in calculations with the harmonic oscillator potential. The existence of the uncertain ex- cited state with $J=2^{-}, T=1$ has been discussed theoretically, and the theoretical results confirm its existence.

We thank Prof. Mouna Bouhelal (Laboratoire de Physique Applique et Theorique, Universite Labri Tebessi, Tebessa, Algeria) for providing us with the PSDPF interaction and for the professional discussions.

1. P.J. Davies et al. Toward the limit of nuclear binding on the $N=Z$ line: Spectroscopy of ${ }^{96} \mathrm{Cd}$. Phys. Rev. C 99, 021302 (2019).

2. Eunja Ha, Myung-Ki Cheoun, H. Sagawa. Effects of the Coulomb and the spin-orbit interaction in a deformed mean field on the pairing correlations in $N=Z$ nuclei. Phys. Rev. C 99, 064304 (2019).

3. T. Inakura, S. Mizutori. Rod-shaped rotational states in $N=Z$ even-even nuclei from ${ }^{12} \mathrm{C}$ to ${ }^{32} \mathrm{~S}$. Phys. Rev. $C$ 98, 044312 (2018).

4. H. Morita, Y. Kanada-En'yo. Low-energy Gamow-Teller transitions in deformed $N=Z$ odd-odd nuclei. Phys. Rev. C 98, 034307 (2018).

5. E. Ha, M.-K. Cheoun, H. Sagawa. Spin singlet and spin triplet pairing correlations on shape evolution in sd-shell $N=Z$ Nuclei. Phys. Rev. C 97, 024320 (2018).

6. K. Kaneko, Y. Sun, G. de Angelis. Enhancement of highspin collectivity in $N=Z$ nuclei by the isoscalar neutronproton pairing. Nucl. Phys. A 957, 144 (2017).

7. I. Wiedenhöver, A.H. Wuosmaa, R.V.F. Janssens, C.J. Lister, M.P. Carpenter, H. Amro, P. Bhattacharyya, B.A. Brown, J. Caggiano, M. Devlin, A. Heinz, F.G. Kondev, T. Lauritsen, D.G. Sarantites, S. Siem, L.G. Sobotka, A. Sonzogni. Identification of the $I^{\pi}=10^{+}$yrast rotational state in ${ }^{24} \mathrm{Mg}$. Phys. Rev. Lett. 87, 142502 (2001).

8. I. Wiedenhöver, A.H. Wuosmaa, R.V.F. Janssens, C.J. Lister, M.P. Carpenter, Janssens, R.V.F. Amro, H. Caggiano, J. Heinz, A. Kondev, F.G. Lauritsen, T. Siem, S. Sonzogni, A. Bhattacharyya, P. Devlin, M. Sarantites, D.G. Sobotka. Measurement and analysis of quadruple $(\alpha \gamma \gamma)$ angular correlations for high spin states of ${ }^{24} \mathrm{Mg}$. Nucl. Phys. A 682, 22 (2001).

9. E.S. Diffenderfer, L.T. Baby, D. Santiago-Gonzalez, N. Ahsan, A. Rojas, A. Volya, I. Wiedenhöver, A.H. Wuosmaa, M.P. Carpenter, R.V.F. Janssens, C.J. Lister, M. Devlin, D.G. Sarantites, L.G. Sobotka, Y. Utsuno, M. Horoi. Highspin spectrum of ${ }^{24} \mathrm{Mg}$ studied through multiparticle angular correlations. Phys. Rev. C 85, 034311 (2012).

10. M. Bouhelal, F. Haas, E. Caurier, F. Nowacki, A. Bouldjedrib. Negative-parity intruder states of the neutron-rich $N=20, Z=14-16$ isotones: a $1 \hbar \omega$ shell model description. Acta. Phys. Pol. B 40, 639 (2009).

11. P. Marley, D.G. Jenkins, P.J. Davies, A.P. Robinson, R. Wadsworth, C.J. Lister, M.P. Carpenter, R.V.F. Jan- 
ssens, C.L. Jiang, T.L. Khoo, T. Lauritsen, D. Seweryniak, S. Zhu, S. Courtin, F. Haas, D. Lebhertz, M. Bouhelal, J.C. Lighthall, A.H. Wuosmaa, D. O'Donnell. Highresolution spectroscopy of decay pathways in the ${ }^{12} \mathrm{C}\left({ }^{12} \mathrm{C}\right.$, $\gamma$ ) reaction. Phys. Rev. C 84, 044332 (2011).

12. D.G. Jenkins, M. Bouhelal, S. Courtin, M. Freer, B.R. Fulton, F. Haas, R.V.F. Janssens, T.L. Khoo, C.J. Lister, E.F. Moore, W.A. Richter, B. Truett, A.H. Wuosmaa. $\gamma$ ray spectroscopy of the $A=23, T=1 / 2$ nuclei ${ }^{23} \mathrm{Na}$ and ${ }^{23} \mathrm{Mg}$ : High-spin states, mirror symmetry, and applications to nuclear astrophysical reaction rates. Phys. Rev. C 87, 064301 (2013).

13. M. Bouhelal, F. Haas, E. Caurier, F. Nowacki, A. Bouldjedri. PSDPF interaction to describe the $1 \hbar \omega$ intruder states in sd shell nucleiNucl. Phys. A 864, 113 (2011).

14. M. Bouhelal, F. Haas, E. Caurier, F. Nowacki, A. Bouldjedri. PSDPF interaction to describe the $1 \hbar$ ? intruder states in sd shell nucleiNucl. Phys. Rev. C 96, 044304 (2017).

15. R. S. Lubna, Vandana Tripathi, S.L. Tabor, P.-L. Tai, K. Kravvaris, P.C. Bender, A. Volya, M. Bouhelal, C.J. Chiara, M.P. Carpenter, R.V.F. Janssens, T. Lauritsen, E.A. McCutchan, S. Zhu, R.M. Clark, P. Fallon, A.O. Macchiavelli, S. Paschalis, M. Petri, W. Reviol, D.G. Sarantites. Intruder configurations of excited states in the neutron-rich isotopes ${ }^{33} \mathrm{P}$ and ${ }^{34} \mathrm{P}$. Phys. Rev. C 97, 044312 (2018).

16. S. Aydin, M. Ionescu-Bujor, G.Tz. Gavrilov, B.I. Dimitrov, S.M. Lenzi, F. Recchia, D. Tonev, M. Bouhelal, F. Kavillioglu, P. Pavlov, D. Bazzacco, P.G. Bizzeti, A.M. Bizzeti-Sona, G. de Angelis, I. Deloncle, E. Farnea, A. Gadea, A. Gottardo, N. Goutev, F. Haas, T. Huyuk, H. Laftchiev, S. Lunardi, Tz.K. Marinov, D. Mengoni, R. Menegazzo, C. Michelagnoli, D.R. Napoli, P. Petkov, E. Sahin, P.P. Singh, E.A. Stefanova, C.A. Ur, J.J. Valiente-Dobón, M.S. Yavahchova. High-spin states and lifetimes in ${ }^{33} \mathrm{~S}$ and shell-model interpretation in the $s d-f p$ space. Phys. Rev. C 96, 024315 (2017).

17. B. Fu, M. Seidlitz, A. Blazhev, M. Bouhelal, F. Haas, P. Reiter, K. Arnswald, B. Birkenbach, C. Fransen, G. Friessner, A. Hennig, H. Hess, R. Hirsch, L. Lewandowski, D. Schneiders, B. Siebeck, T. Steinbach, T. Thomas, A. Vogt, A. Wendt, K. Wolf, K.O. Zell. $\gamma$-ray spectroscopy of ${ }^{33} \mathrm{P}$ and ${ }^{33} \mathrm{~S}$ after fusion-evaporation reactions. Phys. Rev. C 94, 034318 (2016).

18. A. Radhi, Ali A. Alzubadi, A.H. Ali. Magnetic dipole moments, electric quadrupole moments, and electron scattering form factors of neutron-rich sd- pf cross-shell nuclei. Phys. Rev. C 97, 064312 (2018).

19. B.A. Brown, A. Etchegoyen, W.D. Rae, N.S. Godwin, W.A. Richter, C.H. Zimmerman, W.E. Ormand, J.S. Winfield. $M S U-N S C L$ Report No. 524, 1985 (unpublished).

20. S. Cohen, D. Kurath. Spectroscopic factors for the $1 p$ shell. Nucl. Phys. A 101, 1 (1967).

21. B.A. Brown, W.A. Richter. New "USD" Hamiltonians for the sd shell. Phys. Rev. C 74, 034315 (2006).
22. E.K. Warburton, B.A. Brown. Effective interactions for the 0p1s0d nuclear shell-model space. Phys. Rev. C 46, 923 (1992).

23. S. Nummela, P. Baumann, E. Caurier, P. Dessagne, A. Jokinen, A. Knipper, G. Le Scornet, C. Miehé, F. Nowacki, M. Oinonen, Z. Radivojevic, M. Ramdhane, G. Walter, J. Äystö. Spectroscopy of ${ }^{34 ? 35} \mathrm{Si}$ by $\beta$ decay: $s d-f p$ shell gap and single-particle states. Phys. Rev. C 63, 044316 (2001).

24. A.A. Al-Sammarraie, F.I. Sharrad, H.A. Kassim. Nuclear structure for ${ }^{24} \mathrm{Mg}$ within sd-shell model space Hamiltonians. Armenian Journal of Physics 8, 170 (2015).

25. R.E. Tribble, G.T. Garvey, J.R. Comfort. Particle-hole nature of the two lowest 3 -states in ${ }^{24} \mathrm{Mg}$. Phys. Lett. B 44, 366 (1973).

26. http://www.nndc.bnl.gov/

27. R.B. Firestone. Nuclear Data Sheets for $A=24$. Nucl. Data Sheets 108, 2319 (2007).

28. F.I. Sharrad. Binding energy and B(E2; $\left.4_{1}^{+} \rightarrow 2_{1}^{+}\right)$calculations for nuclei with $A=20$. AIP Conf. \& Proc. 1528, 376 (2013).

29. H. Zarek, S. Yen, B.O. Pich, T.E. Drake, C. F. Williamson, S. Kowalski, C.P. Sargent. Inelastic electron scattering to negative-parity states of ${ }^{24} \mathrm{Mg}$. Phys. Rev. C 29, 1664 (1984).

30. A.A. Al-Sammarraie, M.L. Inche Ibrahim, M.A. Saeed, F.I. Sharrad, H.A. Kassim. Inelastic electric and magnetic electron scattering form factors of ${ }^{24} \mathrm{Mg}$ nucleus: Role of g-factors. Int. J. Mod. Phys. E 26, 1750032 (2017).

31. B.A. Brown. Lecture Notes in Nuclear Structure Physics (Michigan State Univ., 2011).

32. A.A. Al-Sammarraie, F.I. Sharrad, N. Yusof, H.A. Kassim. Longitudinal and transverse electron-nucleus scattering form factors of ${ }^{25} \mathrm{Mg}$. Phys. Rev. C 92, 034327 (2015).

33. B.A. Brown, B.H. Wildenthal, C.F. Williamson, F.N. Rad, S. Kowalski, Hall Crannell, J.T.O' Brien. Shell-model analysis of high-resolution data for elastic and inelastic electron scattering on ${ }^{19}$ F. Phys. Rev. C 32, 1127 (1985).

34. H. Chjandra, G. Sauer. Relativistic corrections to the elastic electron scattering from ${ }^{208} \mathrm{~Pb}$. Phys. Rev. C 13, 245 (1976).

35. L.J. Tassie, F.C. Barker. Application to electron scattering of center-of-mass effects in the nuclear shell model. Phys. Rev. 111, 940 (1958).

36. D. Branford, N. Gardner, I.F. Wright. Evidence for negative-parity rotational bands in ${ }^{24} \mathrm{Mg}$. Phys. Lett. 36, 456 (1971).

37. A. Johnston, T.E. Drake. A study of ${ }^{24} \mathrm{Mg}$ by inelastic electron scattering. J. Phys. A: Math., Nucl. Gen. 7, 898 (1974) (un available online).

38. B.T. Lawergren, A.T.G. Fergusono, G.C. Morrision. States with $T=1$ in ${ }^{20} \mathrm{Ne},{ }^{24} \mathrm{Mg}$ and ${ }^{28} \mathrm{Si}$. Nucl. Phys. A 108, 325 (1968).

Received 06.05.20 
А.А. Ель-Саммарі, Ф А.Ахмед, А.А. Охунов

РОЗРАХУНКИ СТРУКТУРИ

СТАНІВ НЕГАТИВНОЇ ПАРНОСТІ ЯДРА ${ }^{24} \mathrm{Mg}$

В ОБОЛОНКОВІЙ МОДЕЛІ З БАЗИСОМ ВЕЛИКОЇ ВИМІРНОСТІ

Досліджено стани з негативною парністю ядра ${ }^{24} \mathrm{Mg}$ в рамках оболонкової моделі. Ми грунтуємось на розрахунках рівнів енергії, квадратів повних форм-факторів та ймовірностей переходів і використовуємо p-sd-pf (PSDPF) гамільтоніан у великому базисі моделі $(0+1) \hbar \omega$. Порівняння експериментальних та теоретичних станів показало добре узгодження в рамках урізаного модельного простору. Розрахун- ки на основі PSDPF гамільтоніана успішно відтворили дані для квадратів повних форм-факторів та ймовірностей переходів для станів негативної парності в ядрі ${ }^{24} \mathrm{Mg}$. Ці величини залежать від одночастинкових елементів матриці густини, які отримані з PSDPF гамільтоніана. Хвильові функції елементів радіальної одночастинкової матриці, що розраховані з використанням потенціалу гармонічного осцилятора, підходять для прогнозування експериментальних даних шляхом внесення поправок, пов'язаних із рухом центра мас системи.

Ключові слова: p-sd-pf (PSDPF), стани 3 негативною парністю, розсіювання електронів, оболонкова модель, форм-фактор, ядро ${ }^{24} \mathrm{Mg}$. 